\title{
Meta-Analysis of CSF Diversion Procedures and Dural Venous Sinus Stenting in the Setting of Medically Refractory Idiopathic Intracranial Hypertension
}

S.R. Satti, L. Leishangthem, and M.I. Chaudry E

\begin{abstract}
BACKGROUND AND PURPOSE: In medically refractory idiopathic intracranial hypertension, optic nerve sheath fenestration or CSF shunting is considered the next line of management. Venous sinus stenosis has been increasingly recognized as a treatable cause of elevated intracranial pressure in a subset of patients. In this article, we present the results of the largest meta-analysis of optic nerve sheath fenestration, CSF shunting, and dural venous sinus stenting. This is the only article that compares these procedures, to our knowledge.
\end{abstract}

MATERIALS AND METHODS: We performed a PubMed search of all peer-reviewed articles from 1988 to 2014 for patients who underwent a procedure for medically refractory idiopathic intracranial hypertension.

RESULTS: Optic nerve sheath fenestration analysis included 712 patients. Postprocedure, there was improvement of vision in $59 \%$, headache in $44 \%$, and papilledema in $80 \%$; $14.8 \%$ of patients required a repeat procedure with major and minor complication rates of $1.5 \%$ and $16.4 \%$, respectively. The CSF diversion procedure analysis included 435 patients. Postprocedure, there was improvement of vision in $54 \%$, headache in $80 \%$, and papilledema in $70 \% ; 43 \%$ of patients required at least 1 additional surgery. The major and minor complication rates were $7.6 \%$ and $32.9 \%$, respectively. The dural venous sinus stenting analysis included 136 patients. After intervention, there was improvement of vision in $78 \%$, headache in $83 \%$, and papilledema in $97 \%$ of patients. The major and minor complication rates were $2.9 \%$ and $4.4 \%$, respectively. Fourteen additional procedures were performed with a repeat procedure rate of $10.3 \%$. Three patients had contralateral stent placement, while 8 had ipsilateral stent placement within or adjacent to the original stent. Only 3 patients required conversion to CSF diversion or $2.2 \%$ of patients with stents.

CONCLUSIONS: Patients with medically refractory idiopathic intracranial hypertension have traditionally undergone a CSF diversion procedure as the first intervention. This paradigm may need to be re-examined, given the high technical and clinical success and low complication rates with dural venous sinus stenting.

ABBREVIATIONS: $\mathrm{BMI}=$ body mass index; $I I \mathrm{H}=$ idiopathic intracranial hypertension; ONSF = optic nerve sheath fenestration

diopathic intracranial hypertension (IIH), previously referred to as pseudotumor cerebri and benign intracranial hypertension, is a syndrome defined by elevated intracranial hypertension without radiographic evidence of a mass lesion in the brain. ${ }^{1}$ The overall prevalence of IIH in North America has been estimated to

Received December 10, 2014; accepted after revision March 3, 2015.

From the Department of Neurointerventional Surgery (S.R.S., L.L.), Christiana Care Health Center, Wilmington, Delaware; and Department of Neuroradiology (M.I.C.), Medical University of South Carolina, Charleston, South Carolina.

Please address correspondence to Sudhakar R. Satti, MD, 4755 Ogletown-Stanton Rd, Newark, DE 19718; e-mail: ssatti@christianacare.org

Indicates article with supplemental on-line tables.

Evidence-Based Medicine Level 1.

http://dx.doi.org/10.3174/ajnr.A4377 be $0.9-1.07 / 100,000^{2,3}$; however, in women with obesity between 20 and 44 years of age, the prevalence rises to $15-19 / 100,000 .^{2}$

Although headache is the most common presenting symptom, seen in $92 \%-94 \%$ of patients, ${ }^{4,5} \mathrm{IIH}$ also represents a significant cause of chronic headaches. In some patients, there may be vision changes, ${ }^{6-9}$ which, if not corrected, may progress to permanent visual loss. ${ }^{10,11}$

The standard medical treatment includes weight loss, acetazolamide, diuretics, and repeat high-volume lumbar punctures. In patients with medically refractory IIH or progressive visual loss, a CSF-diversion procedure (lumboperitoneal shunt, ventriculoperitoneal shunts, or optic nerve sheath fenestration) is considered the next line of management. ${ }^{9,12}$

CSF diversion procedures in the setting of medically refractory IIH have been described in the literature dating back to 1955, by 
Jackson and Snodgrass. ${ }^{13,14}$ These studies are level 3 evidence, comprising case series and individual case reports. There are no prospective randomized controlled studies on lumboperitoneal shunt, ventriculoperitoneal shunts, or optic nerve sheath fenestration, to our knowledge.

Venous sinus stenosis has been increasingly recognized as a treatable cause of elevated intracranial pressure. Venous sinus stent placement was first described by Higgins et al. ${ }^{15}$ During the past 20 years, an increasing number of case reports and larger case series have described dural venous sinus stent placement, and reported high rates of technical success and favorable clinical outcomes. $^{6,7,16-21}$

In this article, we present the results of the largest meta-analysis of optic nerve sheath fenestration, CSF diversion procedure, and venous sinus stent placement for medically refractory $\mathrm{IIH}$ from 1988 to present. We then compare these interventions with a focus on symptom improvement, complications, and the need for repeat procedures.

\section{MATERIALS AND METHODS \\ Search Criteria}

We performed a PubMed search of all peer-reviewed articles from 1988 to date with a combination of key words including "Idiopathic Intracranial hypertension," "Pseudotumor Cerebri" and "Benign Intracranial Hypertension" and "Lumboperitoneal Shunts," "Ventriculoperitoneal Shunts," and "Optic Nerve Sheath Decompression" and "Intracranial Venous Shunts." References from the articles that were identified in the initial search were also reviewed for extraction of additional studies.

\section{Eligibility Criteria}

Studies reporting patients with $\mathrm{IIH}$ and other secondary causes of increased intracranial pressure were separated into 2 groups, and only the patients with IIH who had undergone a procedure were included.

Because these studies were conducted with different aims and protocols, some data on visual acuity, visual fields, CSF pressure, and mean body mass index (BMI) were not available. We attempted to standardize the data as much as possible. The means, ranges, and percentages were calculated for the combined subset of patients with data available for each parameter or outcome. Patients without adequate data were excluded from the analysis to avoid bias and to produce reliable results. Symptom-resolution analysis was performed for only those patients with reported data. Symptom resolution and improvement are presented as improvement, except in the optic nerve sheath fenestration (ONSF) analysis, due to lack of homogeneity of the data. In dural venous sinus stenting and CSF flow-diversion articles, visual acuity and visual fields were combined and described as visual acuity changes.

\section{Exclusion}

All studies written before 1988; series with $<9$ patients for venous sinus stent placement, $<6$ for ONSF, and $<7$ for CSF flow diversion; non-English articles; and reports with inadequate information regarding patient selection and follow-up were excluded.

\section{Data Extraction}

Effort was made to standardize the data for ease of comparison among treatment modalities with a focus on the following end points:

- Presenting symptoms and patient characteristics (BMI and CSF opening pressure).

- Resolution of symptoms (including headache and visual loss).

- Resolution of papilledema.

- Revision rates of the CSF flow-diversion procedures.

- Complication rates.

- Technical success.

In the analysis of CSF-diversion procedures, patients needing revisions were considered as having complications because they needed repeat procedures, though indications were variable.

\section{RESULTS}

\section{Optic Nerve Sheath Fenestration}

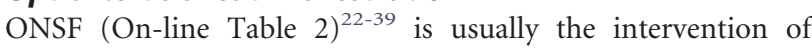
choice for patients with medically refractory IIH presenting with severe visual loss but minimal headache. The procedure has been shown to rapidly improve visual function (both visual acuity and visual fields) and stabilize the visual changes, thus preventing progression. ${ }^{9,28}$

Our PubMed search identified 18 clinical studies with patients undergoing ONSF, including 712 patients and 1153 eyes. The mean age at presentation was 32.4 years (range, $4.4-74$ years), and the mean follow-up period was 21 months (range, 0-160 months).

Sixty percent of patients presented with headaches, 92\% presented with visual acuity changes, and $86 \%$ presented with visual field change. Females represented 82\% (391/476 patients). Unilateral surgeries (53\%, 252/476 patients) were slightly more common than bilateral surgeries. Eighty-nine percent of patients (389/439) had ONSF as their first surgical procedure for medically refractory IIH.

After optic nerve sheath fenestration, visual acuity improved in 59\% (152/257 eyes) and improved or remained stable in $95 \%$ (1011/1066 eyes). Visual fields improved in 68\% (470/688 eyes). Headache improved in $44 \%$ (56/127 patients), and papilledema improved in $80 \%$ (76/95 patients).

In the total 712 patients who underwent ONSF, there were 128 documented complications. Complications included diplopia (55; $43 \%)$, pupillary complications $(32 ; 25 \%)$, late failure (13; $10 \%$ ), and dellen (shallow excavations along the outer edge of the cornea caused by localized dehydration) $(8 ; 6 \%)$.

The total average complication rate (including major and minor complications) was $18 \%$ (128/712). We defined major complications as esotropia, exotropia, retrobulbar hemorrhage, orbital hematoma, orbital apex syndrome, orbital cellulitis, and traumatic optic neuropathy. The rate of major complications was $1.5 \%$ based on the total number of patients in the study group, 712. We defined minor complications as diplopia, late failure, dellen, synechiae, atonic pupil, tonic pupil, anisocoria, pupillary dysfunction, perilimbal conjunctival bleb, peripapillary hemorrhages, disc hemorrhage, cyst formation, and conjunctival abscess. The rate of minor complications was $16.4 \%$. 
On the basis of the total number of procedures performed, $14.86 \%$ required a repeat procedure: $9.21 \%$, repeat ONSF and $5.65 \%$, CSF diversion, In the series of Spoor et al, ${ }^{26} 31.4 \%$ (11 of 35 ) of patients with acute presentation required a second procedure (but a total of 16 procedures if both eyes were included in some patients).

Because all the data were not available for all the cases, denominators used in the calculation for percentages differed.

Unfortunately, BMI was only available for 1 study included in the analysis, Gupta et $\mathrm{al}^{35}$ with an average BMI of 24.4. Opening pressures were only available in 2 studies (Thuente et $\mathrm{al}^{32}$ and Gupta et $\mathrm{al}^{35}$ ), with 30 patients and a mean opening pressure of $29.28 \mathrm{~cm} \mathrm{H}_{2} \mathrm{O}$ (range, 13-70 $\mathrm{cm} \mathrm{H}_{2} \mathrm{O}$ ).

\section{CSF Flow Diversion}

Seventeen studies with a CSF-diversion (On-line Table $3)^{8,40-46,48,55-60}$ procedure meeting the inclusion criteria were analyzed, including 435 patients; 85\% (310/363) were females. Sex was not reported in 3 studies (Abubaker et al, ${ }^{46}$ Tulipan et al, ${ }^{43}$ and Rosenberg et $\mathrm{al}^{41}$ ). The mean age was 31.9 years (range, 6 months to 68 years).

The mean follow-up time was 41 months (range, 1-278 months). Eighty-six percent (164/190) of patients had CSF diversion as their first surgery. The mean CSF opening pressure, reported in 120 cases, was $41.4 \mathrm{~mm} \mathrm{Hg}$ (range, 29-60 mm Hg). After the procedure, headache improved in $80 \%$ (231/ 287) of cases. Papilledema improved in 70\% (107/153) of cases. Visual acuity improved after treatment in 54\% (104/193).

Forty-three percent of patients required at least 1 additional surgery. Most surprising, 154 of 435 patients undergoing CSF flow diversion underwent an additional 428 procedures (not reported in 2 studies: Thambisetty et $\mathrm{al}^{47}$ and Tarnaris et $\mathrm{al},{ }^{48}$ ) or 2.78 additional procedures for each failure. These additional procedures were mainly revisions of the shunt. The most common reason for the shunt revision was shunt obstruction (41\%), followed by low-pressure headache (15\%), shunt failure $(11 \%)$, radicular pain $(3 \%)$, and others $(25 \%)$, which included abdominal pain, tonsillar herniation, valve dysfunction, shunt disconnection, shunt malposition, subdural hematoma, and CSF fistula.

The rate of major complications was 7.6\% (33/435). We defined major complications as shunt infection, tonsillar herniation, subdural hematoma, and CSF fistula.

The rate of minor complications was $32.9 \%$ (143/435). We defined minor complications as abdominal pain, valve dysfunction, radicular pain, shunt disconnection, shunt malposition, low pressure headache and/or CSF leak, and catheter migration. In Rosenberg et $\mathrm{al}^{41}$ operative complications (2/37 patients) were not clearly defined.

\section{Venous Stent Placement}

Our meta-analysis of dural venous sinus stent placement (On-line Table 4) ${ }^{6,7,16-21}$ included 8 studies with 136 patients. Eighty-eight percent were female (119/136 cases). The mean age was 34.5 years (range, 10-64 years), and the mean follow-up time was 22.9 months (range, 1-136 months). The mean BMI was $34 \mathrm{~kg} / \mathrm{m}^{2}$ (range, $22-73 \mathrm{~kg} / \mathrm{m}^{2}$ ). The mean CSF pressure was $34.3 \mathrm{~mm} \mathrm{Hg}$ (range, 22-73 $\mathrm{mm} \mathrm{Hg}$ ). Seventy-three percent (88/121 cases) of patients who were medically refractory underwent venous stent placement as the first intervention.

Nearly all of the stents used were self-expanding nitinol stents; however, $<5 \%$ of studies described placement of balloonmounted stents. Stents included the following: Bridge X3 stent (Medtronic, Santa Rosa, California), ${ }^{16}$ S.M.A.R.T. stent (Cordis, Fremont, California), ${ }^{16}$ Complete SE stent (Medtronic), ${ }^{19}$ Zilver biliary stent (Cook Medical, Bloomington, Indiana), ${ }^{6,20,21}$ (Worthington Biochemical, Lakewood, New Jersey), Precise self-expanding stent (Cordis), ${ }^{21}$ or Acculink stent (Guidant, St. Paul, Minnesota). ${ }^{21}$

After the intervention, headache improved in $83 \%(101 / 121$ cases) and papilledema improved in $97 \%$ of patients $(104 / 108$ cases). Visual acuity changes improved in 78\% (40/51 cases) after treatment. The mean prestent pressure gradient was $20.6 \mathrm{~mm} \mathrm{Hg}$ (range, 4-50 $\mathrm{mm} \mathrm{Hg}$ ), and the mean poststent pressure gradient was $2.7 \mathrm{~mm} \mathrm{Hg}$ (range, $0-23 \mathrm{~mm} \mathrm{Hg}$ ). Sixty-seven percent ( $n=$ 83 ) of stents were placed in the right transverse sinus, $29 \%$ ( $n=$ 36) of stents were placed in the left transverse sinus, and $4 \%$ were not specified. Stent location was not reported in 16 cases (Higgins et $\mathrm{al}^{17}$ and Ahmed et $\mathrm{al}^{19}$ ).

Of the 136 patients who underwent stent placement, 7.4\% (10/136 cases) had complications, though no fatalities were reported. The major complication rate was $2.9 \%$ (4/136) and was defined as a subdural hematoma. The minor complication rate was $4.4 \%(6 / 136)$ and was defined as transient hearing loss, femoral pseudoaneurysm, retroperitoneal hematoma, urinary tract infection, and syncope.

Fourteen additional procedures were performed; therefore, the rate of repeat procedures was $10.3 \%$ (14/136). Additional procedures were performed in $8 \%$ of patients. Contralateral stent placement was performed in 3 patients, and additional ipsilateral stent placement at or near the original stent for restenosis, in 8 patients. Conversion to CSF diversion was seen in only 3 patients or $2.2 \%$ of total patients initially receiving stents.

Here again, similar to the ONSF studies, because all data were not available for all the cases, denominators used in calculation for percentages differed.

\section{DISCUSSION}

Idiopathic intracranial hypertension has been described dating back to 1893 , by Quinke, who proposed the term "meningitis serosa." 49,50 The presenting symptoms included headache, blurred vision, and vomiting. The clinical syndrome was later described as "pseudotumor cerebri" by Nonne in $1904^{49}$ and then "benign intracranial hypertension" by Foley in 1955. 10,49,50 The inclusion of "benign" in the description was challenged in 1969 by Buchheit et $\mathrm{al},{ }^{49,51}$ who recognized that papilledema was present in almost $95 \%$ of the patients, which, if left untreated, could progress to optic atrophy and irreversible blindness. The term "idiopathic intracranial hypertension" was introduced in $2011^{50}$ and may be increasingly used because the etiology and pathophysiology are poorly understood.

Idiopathic intracranial hypertension is currently defined by the Modified Dandy Criteria ${ }^{1,9}$ and includes an elevation of intracranial pressure with a normal composition of CSF and no intra- 
cranial mass or venous sinus thrombosis. By using the Modified Dandy's Criteria, patients with a heterogeneous group may potentially be combined, confounding management and outcomes.

The underlying pathophysiology of elevated intracranial pressure can be divided into 2 groups, CSF overproduction and decreased CSF absorption. Some early hypotheses for IIH included serous meningitis resulting in decreased CSF outflow (Quinke), increased CSF in the subarachnoid space (Passot), hydrocephalus due to remote effects of bacterial toxins or otitis media (Warrington), and altered vasomotor control of the intracranial vascular bed (Dandy). ${ }^{52}$

The mainstays of conservative therapy include weight loss, medications to reduce CSF production, and repeated high-volume lumbar punctures. Despite these measures, some patients may have progressive symptoms or develop visual changes. $\mathrm{Pa}-$ tients who fail conservative measures are generally referred for CSF flow-diversion procedures such as lumboperitoneal shunting, ventriculoperitoneal shunting, ${ }^{13,14}$ or optic nerve sheath fenestration. More recently, dural venous sinus stent placement has been described in the literature.

ONSF appears to have the highest success rate in patients with visual field changes or vision loss, 86\%-92\% compared with CSF flow diversion or sinus stent placement, $58 \%$ and $65 \%$, respectively. ONSF was associated with the lowest improvement of headache, $60 \%$, compared with sinus stent placement $(89 \%)$ or CSF flow diversion $(80 \%)$. ONSF was also associated with a low major complication rate of $2 \%$, a minor complication rate of $16 \%$, and a revision rate of $15 \%$. From a practical standpoint, ONSF may not be as readily available, and recovery from the procedure should be considered.

The most common indication for CSF flow-diversion procedures is severe headache or progressive visual change. There was a major complication rate of $8 \%$ and a minor complication rate of $33 \%$ in the 435 patients included. Considering this young population, with an average age of 31.9 , the high reported repeat procedure rate of $43 \%$ is concerning. Most repeat procedures were shunt revisions. Forty-three percent of patients ultimately needed an additional procedure during the average follow-up period of 41 months. Of 358 patients (some patients were excluded in the denominator because of missing data), 154 patients underwent 428 additional procedures, or 2.78 additional procedures in $>43 \%$ of patients undergoing CSF flow diversion.

There is limited but growing literature for dural venous sinus stent placement in the setting of elevated intracranial pressure and dural venous sinus stenosis. Dural venous sinus stenting may cause elevated intracranial pressure by 2 different mechanisms. The first is an increased gradient between the CSF space and the cerebral venous sinuses. A second is decreased CSF absorption by the arachnoid granulations, which are pressure-sensitive. ${ }^{3,12,16,17,20}$ Stent placement has been hypothesized to lower intracranial pressure by removing a Starling-like resistor, thereby removing the positive feedback loop. ${ }^{19}$

With noninvasive imaging, such as contrast-enhanced MRV, 3 patterns of dural venous sinus stenting may be amenable to sinus stent placement:

- Focal stenosis of the superior sagittal sinus.
- Bilateral transverse/sigmoid sinus stenosis.

- Unilateral transverse/sigmoid sinus stenosis with contralateral hypoplasia/aplasia.

Although most patients with stents included in this meta-analysis underwent stent placement as the first intervention for medically refractory IIH, $10.3 \%$ of patients underwent stent placement as a second procedure. Dural venous sinus stenting could be considered in patients who have failed the traditional CSF-diversion procedure.

Given the cost and morbidity of repeat procedures, CSF shunting as the criterion standard treatment should be re-evaluated. Ahmed et $\mathrm{al}^{53}$ reported that in their health care system in Australia, the cost of the initial dural venous sinus stent placement was similar to the cost of the initial CSF flow-diversion procedure; however, when one took into account the cost of revisions, the total cost of CSF flow diversion was 5 times higher. Although CSF flow diversion is commonly performed, the literature regarding outcomes and high rates of revision does not compare favorably with recent data on venous sinus stent placement when using strict patient selection, dual antiplatelet therapy, and modern devices. We would argue that evaluation for underlying venous sinus stenosis should be undertaken with MRV (and confirmed, if present, with conventional catheter-based venography and pressure measurements) before any CSF flow-diversion procedure.

\section{Limitations}

Meta-analysis by definition is limited by a retrospective design; therefore, data collection was inconsistent and/or incomplete for both pre- and postoperative parameters. This was further compounded by inclusion of different surgical modalities and specialists. Ophthalmologists focused on vision, and most ONSF studies lacked CSF pressure and BMI. Neurosurgeons focused on headache, while neurointerventionalists focused on headaches and papilledema. All studies lacked consistent data on visual acuity and field changes. Different studies had different definitions for improvement, indications for surgery, and variable long-term follow-up (1-278 months). Although there were fewer patients included in the dural venous sinus stenting group than in the others, 136 patients who met inclusion should generate reliable data for comparison.

Despite these challenges, we summarized the available data as rigorously as possible, with an emphasis on presenting symptoms, resolution of presenting symptoms, repeat procedure rates, and complication rates.

In the CSF flow-diversion group, some pediatric patients were included because they could not be separated because individual data were not available. ${ }^{8,40,42,44,47,54-56}$ In the ONSF studies by Corbett et $\mathrm{al}^{24}$ and Spoor et $\mathrm{al}^{26}{ }^{26}$ complications were given in terms of eyes and not patients. We considered the number of eyes as the number of patients for calculation consistency. Nithyanandam et $\mathrm{al}^{36}$ reported complications in terms of percentages, which we had to convert.

In studies in which values of certain parameters were marginally available (eg, CSF pressure $>40 \mathrm{~cm}$ of $\mathrm{H}_{2} \mathrm{O}$ in $73 \%$ of cases in the CSF diversion study by El-Saadany et $\mathrm{al},{ }^{57}$ ), values were pre- 
sented as "NR" or not reported in On-line 3 and excluded from the mean CSF pressure.

\section{Future Directions}

Surgery for medically refractory IIH is performed by different specialties; therefore, the data are inconsistent. Future CSF flowdiversion, ONSF, and dural venous sinus stenting studies for IIH should include documentation of the following:

1) Patient characteristics (age, comorbidities, BMI, symptoms, medications).

2) Formal ophthalmologic examination (pre- and postintervention).

3) CSF studies (pre- and postintervention).

4) For dural sinus stent placement, angiography confirming stenosis and pressure gradients.

5) Long-term clinical follow-up and stent patency.

\section{CONCLUSIONS}

Patients with medically refractory idiopathic intracranial hypertension have traditionally undergone CSF flow-diversion procedures as the first intervention. On the basis of our metaanalysis comparing traditional CSF flow-diversion procedures with venous sinus stent placement, this paradigm may need to be re-examined, given high technical and clinical success with low complications and low repeat-procedure rates associated with stent placement compared with traditional surgical interventions.

\section{ACKNOWLEDGMENTS}

We would like to thank Ene O. Belleh, MLS, AHIP (Academy of Health Information Professionals), reference and instruction services librarian at the Christiana Care Medical Library, for her evidence-based research support.

Disclosures: M. Imran Chaudry—UNRELATED: Board Membership: Covidien Scientific Advisory Board; Consultancy: Covidien, MicroVention, Penumbra, Codman and Shurtleff.

\section{REFERENCES}

1. Friedman DI, Jacobson DM. Diagnostic criteria for idiopathic intracranial hypertension. Neurology 2002;59:1492-95

2. Durcan FJ, Corbett JJ, Wall M. The incidence of pseudotumor cerebri: population studies in Iowa and Louisiana. Arch Neurol 1988;45:875-77

3. Puffer RC, Mustafa W, Lanzino G. Venous sinus stenting for idiopathic intracranial hypertension: a review of the literature. $\mathrm{J} \mathrm{Neu-}$ rointerv Surg 2013;5:483-86

4. Francis CE, Quiros PA. Headache management in idiopathic intracranial hypertension. Int Ophthalmol Clin 2014;54:103-14

5. Wall M. The headache profile of idiopathic intracranial hypertension. Cephalalgia 1990;10:331-35

6. Fields JD, Javedani PP, Falardeau J, et al. Dural venous sinus angioplasty and stenting for the treatment of idiopathic intracranial hypertension. J Neurointerv Surg 2013;5:62-68

7. Donnet A, Metellus P, Levrier O, et al. Endovascular treatment of idiopathic intracranial hypertension: clinical and radiologic outcome of 10 consecutive patients. Neurology 2008;70:641-47

8. Eggenberger ER, Miller NR, Vitale S. Lumboperitoneal shunt for the treatment of pseudotumor cerebri. Neurology 1996;46:1524-30

9. Biousse V, Bruce BB, Newman NJ. Update on the pathophysiology and management of idiopathic intracranial hypertension. J Neurol Neurosurg Psychiatry 2012;83:488-94

10. Ball AK, Clarke CE. Idiopathic intracranial hypertension. Lancet Neurol 2006;5:433-42

11. Friedman DI, Jacobson DM. Idiopathic intracranial hypertension. J Neuroophthalmol 2004;24:138-45

12. Galgano MA, Deshaies EM. An update on the management of pseudotumor cerebri. Clin Neurol Neurosurg 2013;115:252-59

13. Vander Ark GD, Kempe LG, Smith DR. Pseudotumor cerebri treated with lumbar-peritoneal shunt. JAMA 1971;217:1832-34

14. Jackson IJ, Snodgrass SR. Peritoneal shunts in the treatment of hydrocephalus and increased intracranial pressure: a 4-year survey of 62 patients. J Neurosurg 1955;12:216-22

15. Higgins JN, Owler BK, Cousins C, et al. Venous sinus stenting for refractory benign intracranial hypertension. Lancet 2002;359:22830

16. Owler BK, Parker G, Halmagyi GM, et al. Pseudotumor cerebri syndrome: venous sinus obstruction and its treatment with stent placement. J Neurosurg 2003;98:1045-55

17. Higgins JN, Cousins C, Owler BK, et al. Idiopathic intracranial hypertension: 12 cases treated by venous sinus stenting. J Neurol Neurosurg Psychiatry 2003;74:1662-66

18. Bussière M, Falero R, Nicolle D, et al. Unilateral transverse sinus stenting of patients with idiopathic intracranial hypertension. AJNR Am J Neuroradiol 2010;31:645-50

19. Ahmed RM, Wilkinson M, Parker GD, et al. Transverse sinus stenting for idiopathic intracranial hypertension: a review of 52 patients and of model predictions. AJNR Am J Neuroradiol 2011;32:1408-14

20. Albuquerque FC, Dashti SR, Hu YC, et al. Intracranial venous sinus stenting for benign intracranial hypertension: clinical indications, technique, and preliminary results. World Neurosurg 2011;75:64852; discussion 592-95

21. Kumpe DA, Bennett JL, Seinfeld J, et al. Dural sinus stent placement for idiopathic intracranial hypertension. J Neurosurg 2012;116:538-48

22. Alsuhaibani AH, Carter KD, Nerad JA, et al. Effect of optic nerve sheath fenestration on papilledema of the operated and the contralateral nonoperated eyes in idiopathic intracranial hypertension. Ophthalmology 2011;118:412-14

23. Brourman ND, Spoor TC, Ramocki JM. Optic nerve sheath decompression for pseudotumor cerebri. Arch Ophthalmol 1988;106:1378-83

24. Corbett JJ, Nerad JA, Tse DT, et al. Results of optic nerve sheath fenestration for pseudotumor cerebri: the lateral orbitotomy approach. Arch Ophthalmol 1988;106:1391-97

25. Sergott RC, Savino PJ, Bosley TM. Modified optic nerve sheath decompression provides long-term visual improvement for pseudotumor cerebri. Arch Ophthalmol 1988;106:1384-90

26. Spoor TC, Ramocki JM, Madion MP, et al. Treatment of pseudotumor cerebri by primary and secondary optic nerve sheath decompression. Am J Ophthalmol 1991;112:177-85

27. Kelman SE, Sergott RC, Cioffi GA, et al. Modified optic nerve decompression in patients with functioning lumboperitoneal shunts and progressive visual loss. Ophthalmology 1991;98:1449-53

28. Kelman SE, Heaps R, Wolf A, et al. Optic nerve decompression surgery improves visual function in patients with pseudotumor cerebri. Neurosurgery 1992;30:391-95

29. Acheson JF, Green WT, Sanders MD. Optic nerve sheath decompression for the treatment of visual failure in chronic raised intracranial pressure. J Neurol Neurosurg Psychiatry 1994;57:1426-29

30. Goh KY, Schatz NJ, Glaser JS. Optic nerve sheath fenestration for pseudotumor cerebri. J Neuroophthalmol 1997;17:86-91

31. Banta JT, Farris BK. Pseudotumor cerebri and optic nerve sheath decompression. Ophthalmology 2000;107:1907-12

32. Thuente DD, Buckley EG. Pediatric optic nerve sheath decompression. Ophthalmology 2005;112:724-27 
33. Knapp CM, Sampath R. Optic nerve sheath fenestration: a five year audit. Neuro-Ophthalmology 2005;29:173-77

34. Chandrasekaran S, McCluskey P, Minassian D, et al. Visual outcomes for optic nerve sheath fenestration in pseudotumour cerebri and related conditions. Clin Experiment Ophthalmol 2006;34:661-65

35. Gupta AK, Gupta A, Kumar S, et al. Endoscopic endonasal management of pseudotumor cerebri: is it effective? Laryngoscope 2007;117:1138-42

36. Nithyanandam S, Manayath GJ, Battu RR. Optic nerve sheath decompression for visual loss in intracranial hypertension: report from a tertiary care center in South India. Indian J Ophthalmol 2008;56:115-20

37. Moreau A, Lao KC, Farris BK. Optic nerve sheath decompression: a surgical technique with minimal operative complications. J Neuroophthalmol 2014;34:34-38

38. Pineles SL, Volpe NJ. Long-term results of optic nerve sheath fenestration for idiopathic intracranial hypertension: earlier intervention favours improved outcomes. Neuro-Ophthalmology 2013;37:12-19

39. Sencer A, Akcakaya MO, Basaran B, et al. Unilateral endoscopic optic nerve decompression for idiopathic intracranial hypertension: a series of 10 patients. World Neurosurg 2014;82:745-50

40. Johnston I, Besser M, Morgan MK. Cerebrospinal fluid diversion in the treatment of benign intracranial hypertension. $J$ Neurosurg 1988;69:195-202

41. Rosenberg ML, Corbett JJ, Smith C, et al. Cerebrospinal fluid diversion procedures in pseudotumor cerebri. Neurology 1993;43:1071-72

42. Burgett RA, Purvin VA, Kawasaki A. Lumboperitoneal shunting for pseudotumor cerebri. Neurology 1997;49:734-39

43. Tulipan N, Lavin PJ, Copeland M. Stereotactic ventriculoperitoneal shunt for idiopathic intracranial hypertension: technical note. Neurosurgery 1998;43:175-76; discussion 176-77

44. Maher CO, Garrity JA, Meyer FB. Refractory idiopathic intracranial hypertension treated with stereotactically planned ventriculoperitoneal shunt placement. Neurosurg Focus 2001;10:E1

45. McGirt MJ, Woodworth G, Thomas G, et al. Cerebrospinal fluid shunt placement for pseudotumor cerebri-associated intractable headache: predictors of treatment response and an analysis of longterm outcomes. J Neurosurg 2004;101:627-32

46. Abubaker K, Ali Z, Raza K, et al. Idiopathic intracranial hypertension: lumboperitoneal shunts versus ventriculoperitoneal shunts_case series and literature review. $B r \quad J$ Neurosurg 2011;25:94-99

47. Thambisetty M, Lavin PJ, Newman NJ, et al. Fulminant idiopathic intracranial hypertension. Neurology 2007;68:229-32

48. Tarnaris A, Toma $\mathrm{AK}$, Watkins $\mathrm{LD}$, et al. Is there a difference in outcomes of patients with idiopathic intracranial hypertension with the choice of cerebrospinal fluid diversion site: a single centre experience. Clin Neurol Neurosurg 2011;113:477-79

49. Pearce JM. From pseudotumour cerebri to idiopathic intracranial hypertension. Pract Neurol 2009;9:353-56

50. Degnan AJ, Levy LM. Pseudotumor cerebri: brief review of clinical syndrome and imaging findings. AJNR Am J Neuroradiol 2011;32:1986-93

51. Buchheit WA, Burton C, Haag B, et al. Papilledema and idiopathic intracranial hypertension. N Engl J Med 1969;280:938-42

52. Bandyopadhyay S. Pseudotumor cerebri. Arch Neurol 2001;58: 1699-701

53. Ahmed RM, Zmudzki F, Parker GD, et al. Transverse sinus stenting for pseudotumor cerebri: a cost comparison with CSF shunting. AJNR Am J Neuroradiol 2014;35:952-58

54. Bynke G, Zemack G, Bynke H, et al. Ventriculoperitoneal shunting for idiopathic intracranial hypertension. Neurology 2004;63:131416

55. Abu-Serieh B, Ghassempour K, Duprez T, et al. Stereotactic ventriculoperitoneal shunting for refractory idiopathic intracranial hypertension. Neurosurgery 2007;60:1039-43; discussion 1043-44

56. Yadav YR, Parihar V, Agarwal M, et al. Lumbar peritoneal shunt in idiopathic intracranial hypertension. Turk Neurosurg 2012;22: $21-26$

57. El-Saadany WF, Farhoud A, Zidan I. Lumboperitoneal shunt for idiopathic intracranial hypertension: patients' selection and outcome. Neurosurg Rev 2012;35:239-43; discussion 243-44

58. Woodworth GF, McGirt MJ, Elfert P, et al. Frameless stereotactic ventricular shunt placement for idiopathic intracranial hypertension. Stereotact Funct Neurosurg 2005;83:12-16

59. Sinclair AJ, Kuruvath S, Sen D, et al. Is cerebrospinal fluid shunting in idiopathic intracranial hypertension worthwhile? A 10-year review. Cephalalgia 2011;31:1627-33

60. Huang LC, Winter TW, Herro AM, et al. Ventriculoperitoneal shunt as a treatment of visual loss in idiopathic intracranial hypertension. J Neuroophthalmol 2014;34:223-28 